\title{
LAS TIC, UNA APUESTA PARA LA ENSEÑANZA DE LAS MATEMÁTICAS Y LA FÍSICA EN LA EDUCACIÓN MEDIA'
}

\author{
María del Pilar Rodríguez Plaza, Gloria Stella Zambrano Cerón y Bryan Camilo Hernández \\ Semillero de Investigación de las Tecnología del Aprendizaje y del Conocimiento (SITAC) \\ Grupo de Investigación en Pedagogía GIP \\ Institución Universitaria Antonio José Camacho \\ Recepción: 01/07/2019. Aceptado: 08/10/2019. \\ Cómo citar este artículo: \\ Rodríguez-Plaza, M.P., Zambrano-Cerón, G.E. y Hernández, B.C. (2019). Las Tic, una apuesta para la enseñanza de las \\ matemáticas y la física en la educación media. Revista Sapientía, 11 (22), 17-27.
}

\section{RESUMEN}

Este artículo pretende mostrar el desarrollo de una experiencia investigativa que logró como producto final el diseño de una página web con contenidos pedagógicos y curriculares para la enseñanza de la matemática y la física en los grados 10 y 11 de bachillerato del colegio Santa Mariana de Jesús de Cali. El sitio web se denomina Mate club ${ }^{2}$. La experiencia investigativa partió de la necesidad académica de estudiantes y profesores para mejorar el rendimiento académico que se presentaba al interior del aula. Los resultados de la investigación fueron obtenidos a partir del método cuantitativo para identificar los avances de los aprendizajes que iban alcanzando los estudiantes en el periodo escolar del año 2018. Los resultados mostraron que hubo avances en el rendimiento académico en las áreas de matemática y física en los distintos grados una vez fue implementada la estrategia Mate Club dentro del aula.

\section{PALABRAS CLAVE}

TIC, enseñanza, matemáticas, física.

\section{ABSTRACT}

This article aims to show the development of a research experience that achieved the final product design of a Web page with pedagogical and curricular contents for the teaching of mathematics and physics in grades 10 and 11 of the Santa Mariana de Jesús Cali high school. The Website is called Mate club. The research experience started from the academic need of students and teachers to improve the academic performance that was presented inside the classroom. The results of the research were obtained from the method of triangulation and longitudinal to identify the progress of the learning that was reaching students in the school year 2018. The results show that there were advances in academic performance in the areas of mathematics and physics in grades 10 and 11, Mate Club was once implemented as a pedagogical resource in the classroom.

\footnotetext{
Esta investigación fue presentada en la "IV Jornada de Experiencias Pedagógicas con TIC en la Formación Docente”, realizada por la Universidad de Playa Ancha de la ciudad Valparaíso-Chile.

2 El sitio web puede consultarse a través del siguiente enlace: https://pilarik0810.wixsite.com/mate-club
} 


\section{KEYWORDS}

TIC, teaching, mathematics, physics.

\section{INTRODUCCIÓN}

Las matemáticas y la física son materias fundamentales en la educación media de cualquier sistema educativo a nivel mundial. Su estudio es importante porque permite el desarrollo de competencias y habilidades de razonamiento y resolución de problemas para ser aplicables a los entornos competitivos que demanda el siglo XXI. Las matemáticas, por ejemplo, tienen una aplicabilidad transversal en las carreras que son consideradas como las carreras del futuro: ingeniería electrónica, ingeniería industrial, ingeniería aeronáutica, ingeniería informática e ingeniería de telecomunicaciones, entre otras; esto según la revista Jiménez (2018) de España.

Pero enseñar las matemáticas o la física como una apuesta para su aplicabilidad en las carreras del futuro requiere que el estudiante de educación media logre superar algunas dificultades que se presentan al momento de ejecutar el pensamiento lógico, la resolución de problemas o la modelación de problemas matemáticos que son habilidades indispensables para abordar también los contenidos de la física. Lo que se encuentra es que todas estas dificultades de aprendizaje del estudiante suelen estar sujetas a la forma de enseñar del docente. Ruiz (2008) expone que los docentes en estas áreas de enseñanza carecen de formación didáctica y en los peores casos no tienen adecuado dominio del tema. Esta situación recae sobre el estudiante con la etiqueta de dificultad en el aprendizaje, cuando, desde la perspectiva del estudiante, y debido al efecto de la poca didáctica del docente las materias son consideradas por ellos como materias difíciles y aburridas. Es decir, la falta de motivación del estudiante por aprender algo está estrechamente vinculada a la manera en que recibe del otro (profesor) los contenidos de enseñanza.
Dicha problemática se vio representada en los grados $10^{\circ}$ y $11^{\circ}$ del colegio Santa Mariana de Jesús de la ciudad de Cali. El desinterés, la escasa participación en clase, el poco compromiso en las tareas y el bajo rendimiento académico hacían parte del panorama diario en las asignaturas de matemáticas y física. Este fenómeno se identificó en los informes educativos realizados por los docentes en el periodo 01 de 2018. Asimismo, en las notas registradas en las rubricas evaluativas del mismo periodo académico escolar.

Para propiciar la participación de los estudiantes en el centro educativo se hizo apertura de espacios de diálogo a través de un grupo focal, donde se les formuló la siguiente pregunta: ¿Cuáles son los obstáculos que consideras que se evidencian en los docentes a la hora de explicar sus clases? A la que dieron respuestas como las ilustradas en la Figura 1.:

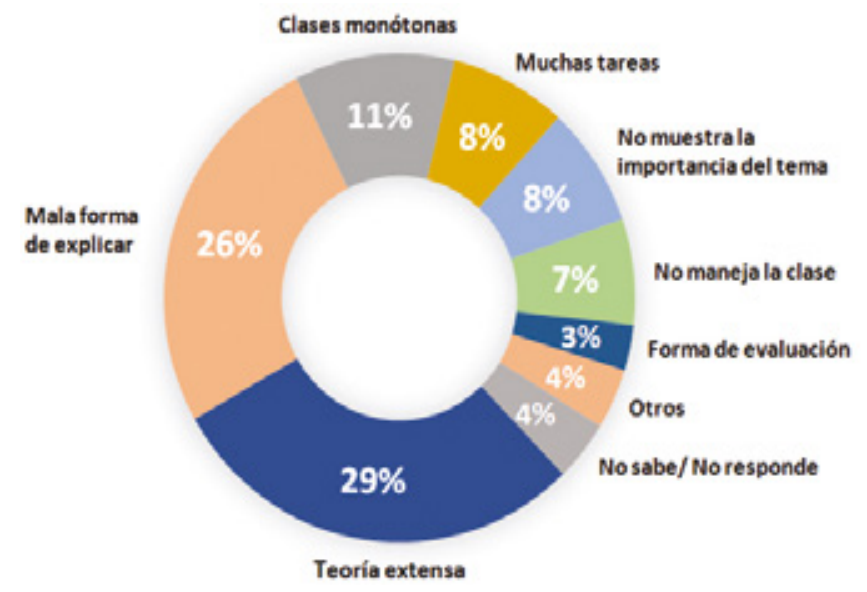

Figura 1. ¿Cuáles son los obstáculos que consideras que se evidencian en los docentes a la hora de explicar sus clases? Fuente: Elaboración propia.

Los resultados presentados en la gráfica se veían claramente representados en la poca motivación de los estudiantes por aprender y aplicar los contenidos curriculares de las asignaturas. Asimismo, tampoco les permitía reconocer su importancia para alcanzar los conocimientos necesarios a fin de lograr un mejor desempeño en las pruebas in- 
ternas y externas, como las de Saber $11^{3}$, lo cual se evidenciaba en los resultados de los simulacros de las pruebas.

El diseño metodológico utilizado en la investigación fue experimental y longitudinal, el cual se expone de manera más detallada en el apartado de la metodología.

La necesidad educativa expuesta llevó a que el equipo investigador formulara y abordara las siguientes preguntas:

¿Cómo mejorar el rendimiento académico de los estudiantes en estas áreas? ¿Cómo mejorar la propuesta de enseñanza de los docentes? ¿Cómo a partir de un recurso tecnológico se pueden favorecer procesos de enseñanza y aprendizaje en estas áreas del saber?

\section{ENSEÑANZA DE LA FÍSICA Y LAS MATEMÁTICAS}

Actualmente hay una exigencia de aplicar propuestas educativas innovadoras en el aula. Sin embargo, la oferta educativa que se ofrece plantea un versus con la educación tradicional, la razón está en la necesidad de ajustar prácticas innovadoras e incluyentes con el fin de ofrecer una educación integral y de calidad. No obstante, la experiencia en el aula evidencia las resistencias que subyacen en algunos docentes para hacer parte del cambio, quizás por el temor a perder su papel protagónico en el proceso educativo o porque su interés por adquirir las competencias para emplear distintos métodos de enseñanza modernos es insuficiente. Punset (citado por Calleja, 2010) afirma que una de las principales causas de la crisis educativa está en que la educación es básicamente la misma a pesar de que el mundo ha cambiado y esto redunda en la falta de interés que tiene el docente para que pueda ser parte de este cambio.

Son las evaluaciones que realiza el estado a los estudiantes que finalizan la Educación Básica.
Ahora bien, el área de matemática no está la excluida del modelo tradicional, gran parte de su contenido suele presentarse de manera repetitiva, poco motivadora y sin establecer relación alguna con las necesidades o vivencias diarias de los educandos, lo que desencadena una serie de situaciones que hacen que el estudio de esta área sea considerado innecesario y carente de sentido.

Por tal motivo, es importante que los docentes construyan estrategias motivadoras para lograr un acercamiento distinto hacia las matemáticas con sus estudiantes, que logré por lo menos generar el deseo por aprenderlas. Pérez y Ramírez (2011) señala que la disposición que presentan los estudiantes para aprender las matemáticas, situar su comprensión y habilidad para usarlas tanto en el planteamiento como en la solución de problemas... dependen de cómo éstas se enseñan en la escuela. Sin embargo, dicho proceso requiere de tiempo, dedicación, esfuerzo y, sobre todo, la actitud con que estas variables se asumen en la práctica pedagógica (Nieves, 1993).

Por su parte la física, aunque es una asignatura cuyas bases teóricas parten de procesos experimentales que necesitan ser estudiados de manera práctica y demostrativa, su estudio presenta deficiencias debido a la dificultad para comprender los conceptos matemáticos implícitos en sus enunciados. Según Hernández, Prada y Gamboa (2016), dentro de las dificultades que manifiestan los alumnos para comprender los enunciados de problemas de Física se encuentran aquellas donde se trata de identificar datos relevantes del problema y cuando se intenta transcribir al lenguaje matemático con dichos datos; estas dificultades surgen por deficiencias en sus habilidades matemáticas.

En este sentido, una de las formas metodológicas para abordar dichas dificultades es la praxis en el laboratorio, en donde el docente ha de identificar la experiencia y conocimientos previos del estudiante para que este adquiera a través de la experimentación un aprendizaje significativo. 
No obstante, dentro de las dificultades para poner en práctica este tipo de metodología está en los costos y en la disposición tanto del docente y la institución para aperturar estos espacios didácticos. Como propuesta y solución al desconocimiento de otros espacios alternativos están los laboratorios PHET Interactive Simulations (también utilizados en Mate Club).

Según Riveros (1995), “el laboratorio se presta para la demostración cuantitativa de datos experimentales, aclara conceptos, verifica leyes o las induce, y es por lo tanto el lugar ideal para aprender a utilizar sus conocimientos en situaciones reales" (p.5).

Por consiguiente, la física vista desde una mirada básicamente teórica con demostraciones matemáticas pasa a ser para el estudiante, una asignatura poco atrayente y con un lenguaje ortodoxo que se dista mucho de la realidad cotidiana en la que vive el sujeto del aprendizaje.

Con lo anterior se puede afirmar que la enseñanza de la física y la matemática van mucho más allá que un impedimento económico. El asunto está más en la disposición que tenga el docente para ser recursivo de acuerdo con su contexto. No se trata de decir que no se requiera apoyo de financiamiento didáctico, se trata también de poner en función la creatividad del docente en los contextos áridos $o$ indiferentes que escatiman y no visibilizan la importancia de invertir en espacios científicos. El deseo por enseñar demostrará la recursividad cognitiva del sujeto de la enseñanza y enseñará a las instituciones y la comunidad académica formas alternativas y por qué no, innovadoras, en las que se pueden aprender la física y la matemática mediante recursos tecnológicos y cotidianos de bajo costo.

\section{LAS TIC EN LOS PROCESOS DE ENSEÑANZA}

En la actualidad, en diversos procesos educativos existen estrategias pedagógicas basadas en la transmisión de habilidades y conocimientos a través de herramientas digitales, por esta razón es importante y necesario que todos los actores que intervienen en estos procesos, profesores, estudiantes e instituciones educativas en general, actúen y participen hacia la proyección del aprendizaje de estas. Es necesario entenderlas como "códigos y dispositivos" que permiten procesar, almacenar y comunicar información en formatos digitales (Vivancos, 2013). Estos formatos digitales pueden contener distinta información de interés, entre ellas información educativa. Sin embargo, se puede encontrar información poco útil que puede como lo refiere Vargas (2015) entorpecer, inclusive dañar el desarrollo del sujeto porque su uso puede suponer cierto facilismo del estudiante.

La realidad es que la incursión de las Tecnologías de la Información y Comunicación (TIC) en el contexto educativo vienen siendo consideradas poco a poco por los docentes como herramientas de apoyo en el diseño e implementación de estrategias pedagógicas mucho más emotivas para los estudiantes. Los docentes se sirven cada vez más de Apps o Webs para dirigir y relacionar los contenidos de sus clases a su praxis de enseñanza, debido a que reconocen que las TIC pueden lograr desde un saber hacer pedagógico emerger habilidades de trabajo cooperativo, autónomo, creativo y de aplicabilidad contextual desde el ritmo propio de aprendizaje de cada estudiante. Ya Arrieta (2013) referirá que las principales características para involucrar mucho más el proceso enseñanza-aprendizaje están sujetas a "la interactividad, la motivación, la autonomía, el papel del alumnado, la cooperación y la comprensión de los contenidos..." (p.8). Pérez (2010) añade otro punto importante, y es que las TIC en el aula tienen su lugar pedagógico en la medida en que respondan a necesidades de formación y de aplicabilidad al contexto vivencial del estudiante.

Pero dichas características no son suficientes, es necesario que el docente sepa cómo emplearlas, de esto dependerá su impacto en el aprendizaje. Por tal motivo se hace necesario preguntarse: ¿cuál es el impacto que voy a lograr como docente en el aula utilizando las TIC? ¿Cuál es la población don- 
de las voy a emplear y cuál es su fin pedagógico? Son preguntas que se evocan a partir de algunos postulados de Bebell (citado en Hermosa del Vasto, 2015).

Si el docente logra volver las TIC un recurso pedagógico podrá potenciar en el estudiante las competencias que propone también la UNESCO en el proyecto ECD-TIC (dirigido esencialmente al profesorado de primaria y secundaria). Estas son:

- Competentes para utilizar tecnologías de la información;

- Buscadores, analizadores y evaluadores de información;

- Solucionadores de problemas y tomadores de decisiones;

- Usuarios creativos y eficaces de herramientas de productividad;

- Comunicadores, colaboradores, publicadores y productores; $y$

- Ciudadanos informados, responsables y capaces de contribuir a la sociedad. (UNESCO, 2008, p.2).

Adicionalmente el uso de las TIC en el aula puede propiciar el interés y motivación del estudiante por su carácter interactivo y visual. Bricall (2000) y Márquez (2002) aseguran que los correos electrónicos y las redes sociales hacen posible un trabajo colaborativo motivacional (Castro, Gusman y Casado, 2007).

Como se observa, las TIC además de ser herramientas pedagógicas, son espacios virtuales con una alta incidencia para la interconectividad con los otros, la interacción con ese otro puede estar para cubrir o emplear un tiempo de ocio o un tiempo pedagógico. De cualquier forma en que se emplee el ambiente virtual, existe una tendencia de sujetos activos y participativos porque el mismo ambiente virtual puede convertirse en un espacio enriquecido que despierte el interés del estudiante. Todo dependerá de la forma en que el docente nutra dicho espacio. Es lo que la UNESCO (2008) llama "estructuración de ambiente de aprendizaje" haciendo un llamado para articular las TIC con diversas pedagogías existentes $y$, sobre todo, hacer de ellas un uso intencional en la enseñanza para fortalecer el aprendizaje significativo del estudiante (Valencia et al, 2016).

El reto está entonces en que el docente plasme su método de enseñanza y por qué no, algunos desafíos que impliquen al estudiante movilizaciones metacognitivas para potenciar el aprendizaje individual y en colectivo. Castro et al (2007) advierte que la creación de entornos formativos implica necesariamente la interacción multidireccional entre los participantes, de esta forma se puede construir el aprendizaje; si dicho objetivo se alcanza se podrá evidenciar la materialidad de productos digitales de los estudiantes. En términos de Bakia, Murphy y Anderson (2011), las competencias académicas de los estudiantes y emotividad con los contenidos de enseñanza mejoran sustancialmente cuando ellos mismos son partícipes de la creación de sus propios contenidos digitales.

No obstante, para que los estudiantes logren elaborar sus propios productos digitales de forma adecuada, es necesario que el docente adquiera la competencia digital que consiste en hacer un uso crítico y seguro de las TIC; además "recuperar, evaluar, almacenar, producir, presentar e intercambiar información, y participar en redes de colaboración a través de Internet" (European Parliament and the Council, 2006, p.15).

Con esto se evidencia que el uso de las TIC en cualquier proceso de enseñanza va mucho más allá de la manipulación o conocimiento técnico de una herramienta tecnológica, implica asumir una posición responsable que le exige al docente empoderarse de su autoaprendizaje para alcanzar y adquirir su competencia digital. 


\section{METODOLOGÍA}

La investigación se realizó a partir de un enfoque cuantitativo; se recogieron datos (notas) del primer período académico a partir de la información entregada por coordinación académica. La información se colocó en contraste con las notas del segundo período académico donde se implementó la apuesta pedagógica Mate Club. Por tal motivo el diseño de estudio fue de carácter experimental y longitudinal donde las variables o medidas repetidas (Rendimiento académico-escalas de valoración) desde la muestra control se seleccionaron en un continuo temporal (dos periodos académicos). Según Hand y Crowder (1996) y Arnau y Bono (2008), los estudios longitudinales sirven para estudiar los procesos de cambio directamente asociados con el paso del tiempo. El cotejo de los datos permitió detallar el avance del rendimiento académico que obtuvieron los estudiantes una vez implementado el sitio web como propuesta pedagógica. El grupo de estudio contó con un total de
54 estudiantes, 22 pertenecientes a grado $10^{\circ}$ y 32 al grado $11^{\circ}$.

Para el diseño del sitio Web se tomaron en consideración las siguientes etapas:

1. Identificación y selección de los contenidos curriculares, actividades evaluativas, material de apoyo y objetivos pedagógicos para el sitio web.

2. Se definieron recursos pedagógicos digitales para que los estudiantes agenciarán un aprendizaje mucho más autónomo en el desarrollo de sus actividades académicas.

3. Se estableció un espacio en el sitio web para la visibilidad y divulgación de los productos finales desarrollados por los estudiantes.

A continuación, se presenta la carta de navegación y contenido de Mate Club:

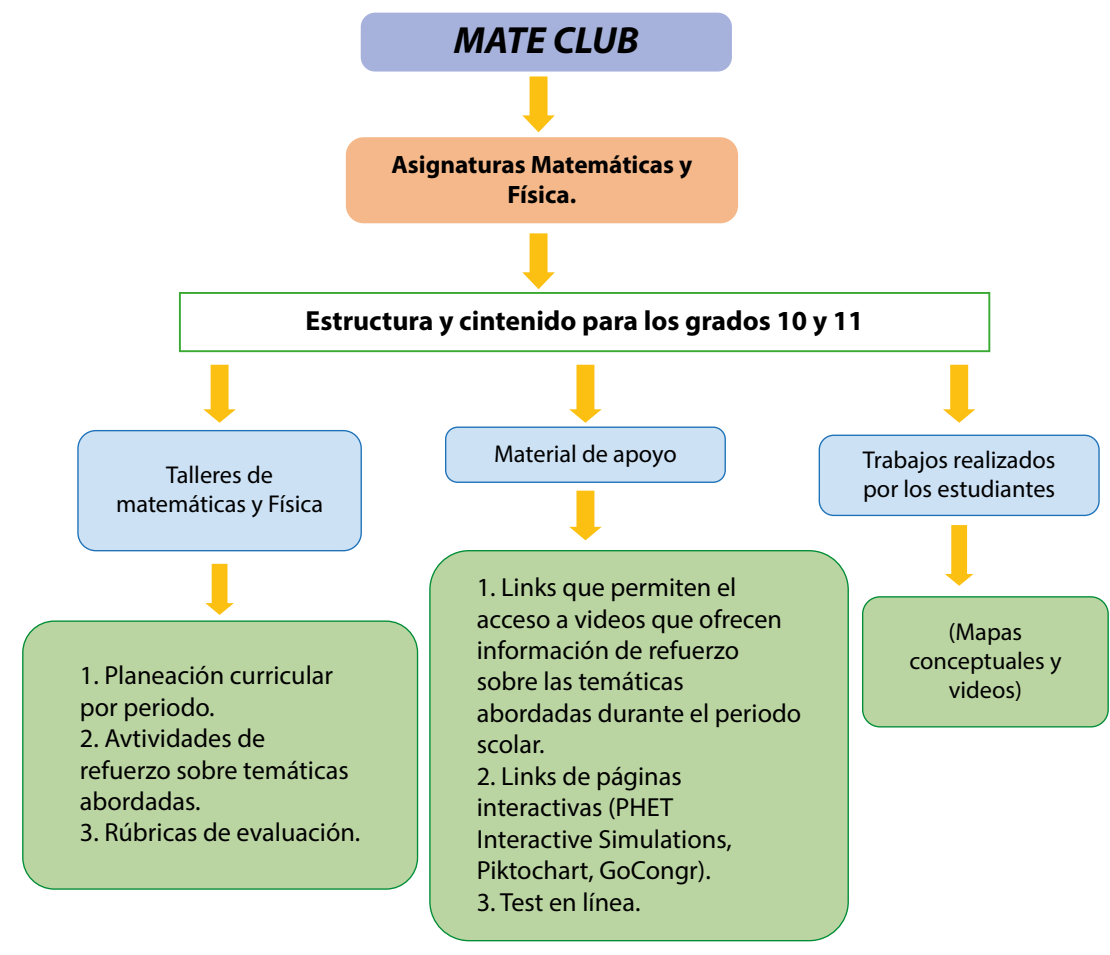

Figura 2. Diagrama sobre la estructura y contenido de MATE CLUB. Fuente: Elaboración propia. 
Como se observa en la Figura 1 el contenido de Mate Club tiene todas las características pedagógicas para realizar una práctica de enseñanza coherente con los objetivos de aprendizaje determinados en cada uno de los grados y asignaturas. Pero, sin duda, el plus de la web está en la posibilidad que tiene el estudiante de visibilizar ante la comunidad educativa y digital su producto final alcanzado en el curso, y de esta forma poder recibir aportes y contribuciones de mejora por parte de sus pares académicos en un espacio alternativo al aula.

\section{RESULTADOS}

En este apartado se muestran registrados de manera estadística los resultados del rendimiento académico obtenido por los estudiantes de acuerdo con los niveles de desempeño bajo, básico, alto y superior, que son los utilizados por la institución educativa y que determinan la posición en la que estos se encuentran de acuerdo con el alcance de las competencias propuestas durante los dos primeros periodos académicos.

Se realizará un análisis de cada gráfica comparando los resultados obtenidos en cada uno de los dos primeros periodos académicos del año escolar 2017-2018.

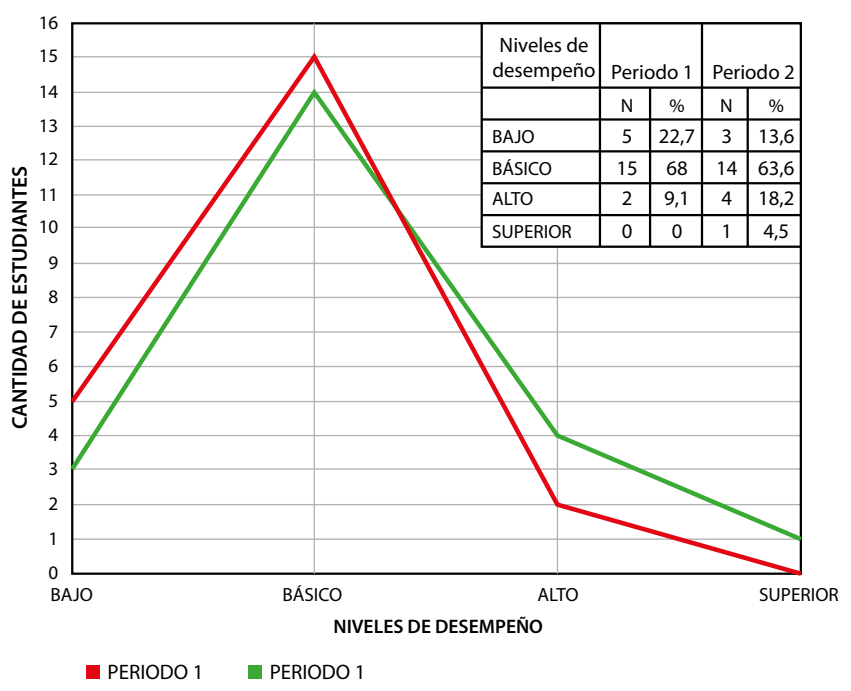

Figura 2. Desempeño académico matemáticas grado 10 primer y segundo periodo. Fuente: Elaboración propia.

En la Figura 2 que corresponde a Matemáticas grado 10, se puede observar que tanto el nivel de desempeño bajo y básico disminuyeron en un 9,1 y un $4,4 \%$ respectivamente, mientras que el desempeño alto y básico aumentaron en un 9,1 y 4,5 $\%$ respectivamente, datos que permiten inferir que aunque en ninguno de los niveles de desempeño el porcentaje tuvo una variación mayor al $10 \%$, la estrategia logró movilizar a los estudiantes hacia una participación activa en cada una de las clases correspondientes a esta asignatura durante el segundo periodo académico.

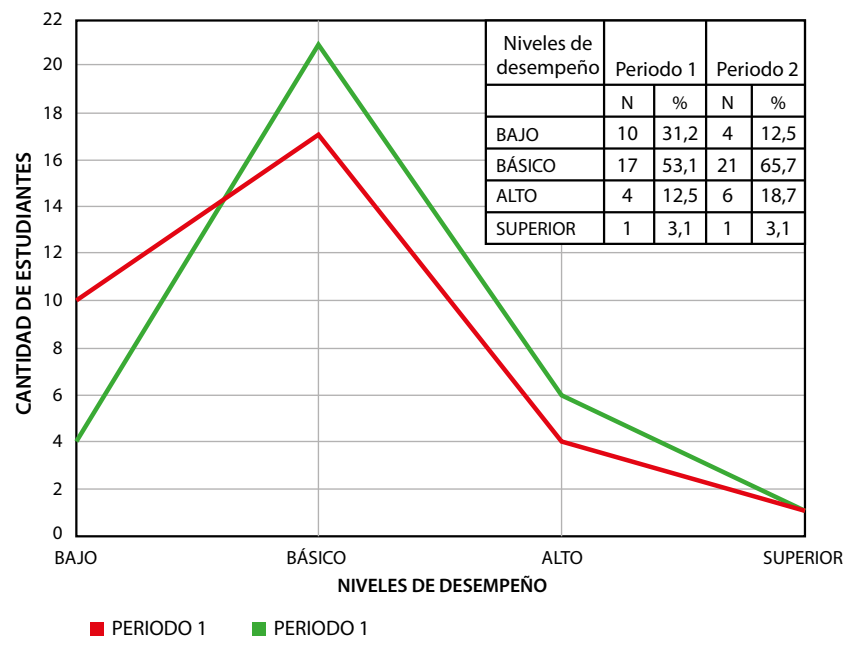

Figura 3. Desempeño académico Matemáticas grado 11 primer y segundo periodo. Fuente: Elaboración propia.

En la Figura 3 se evidencia que en la asignatura de Matemáticas del grado 11 el nivel de desempeño disminuyó en un $18,7 \%$, siendo un avance significativo en el proceso de enseñanza y aprendizaje con los estudiantes del curso y estudiantes que habían reprobado la materia en el primer periodo académico anterior. Al subir el nivel de desempeño, el porcentaje de estudiantes en nivel básico, que es el siguiente en ascenso, aumentó en un $12,6 \%$, haciendo que la mayoría de los estudiantes del curso se concentren en este nivel $(65,7 \%)$. 
Respecto a los niveles de desempeño Alto y Superior, mientras el primero tuvo un aumento del $6,2 \%$ con un alcance no muy relevante, el Superior se mantuvo. Si bien es cierto, aunque en esta asignatura no se percibieron mayores resultados en los últimos niveles de desempeño, se logró disminuir la tasa de reprobación de estudiantes en la asignatura al finalizar el segundo período, hecho que permite inferir que la estrategia generó impacto en sus procesos académicos.

Analizando los resultados obtenidos en Matemáticas para grado 11, se puede observar que la puesta en escena de la estrategia pedagógica usando las TIC en el aula, ofreció alternativas de participación y estudio importantes que permitieron alcanzar mejores niveles de desempeño académico y mayor apropiación de las temáticas abordadas durante las clases.

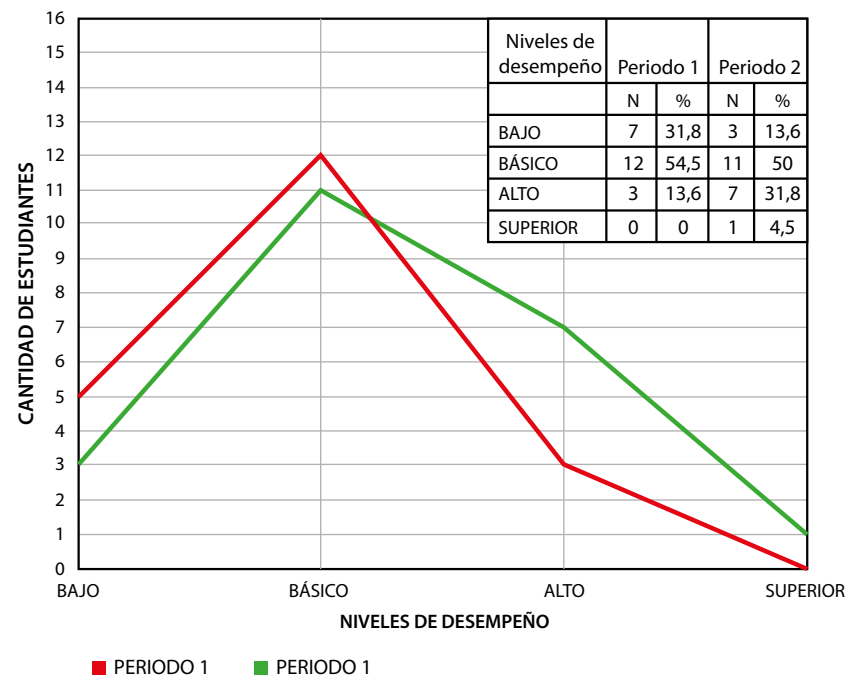

Figura 4. Desempeño académico Física grado 10 primer y segundo periodo. Fuente: Elaboración propia.

En la Figura 4 se puede ver que para la asignatura Física 10, el cambio en los resultados obtenidos al final del segundo periodo, en todos los niveles de desempeño, fueron positivos, especialmente en el Bajo y Alto, ambos con tasa de variación del $18,2 \%$, mientras que para los niveles Básico y Superior su variación fue del $4,5 \%$. A pesar de que aún se encuentra la gran mayoría de estudiantes en un nivel de desempeño básico, se evidencia que la población tiende a aumentar los resultados hacia niveles de desempeños mayores debido a que se evidencia mayor participación y comprensión de las temáticas abordadas en el aula, lo que indica que se están adquiriendo mayores competencias y el aprendizaje se está realizando de manera más autónoma.

Es necesario tener en cuenta que en la medida en que los niveles de desempeño Bajo y Básico disminuyan, se hace más significativo el proceso; se puede observar que la implementación de la estrategia pedagógica en el aula ha permitido que los estudiantes no solamente sientan gusto e interés por aprender, sino que también adquieran mayores competencias.

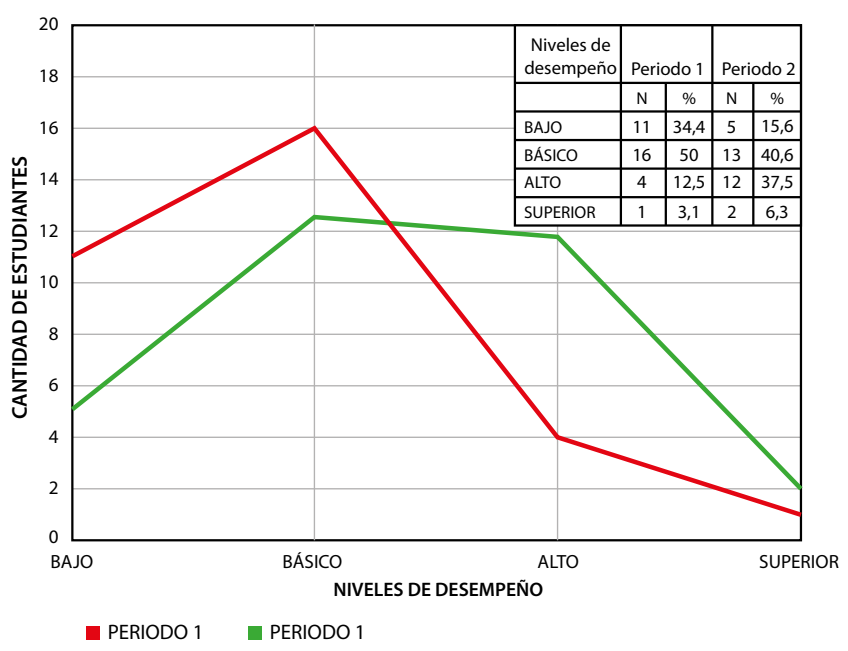

Figura 5. Desempeño académico Física grado 11 primer y segundo periodo. Fuente: Elaboración propia.

En la Figura 5 se observa que el cambio en los resultados obtenidos en el desempeño académico al final del segundo periodo, en la asignatura física 11, fue el más significativo, empezando por la tasa de disminución del nivel de desempeño bajo que alcanzó un 18,74 \%, seguido por el nivel de desempeño básico con una disminución del 9,4\%. Con la tasa de aumento más alta dentro de todo este proceso encontramos al nivel de desempeño Alto que aumentó en un 25\%, haciendo que la mayor 
cantidad de estudiantes del curso se ubicara entre los niveles de desempeño Básico y Alto.

Con estos resultados se puede inferir que la tasa de estudiantes proyectada hacia el nivel de desempeño alto va en aumento. Se resalta el excelente trabajo que realizaron la mayoría de los estudiantes en el aula, su constante participación en clase, el trabajo en equipo y la entrega a tiempo de sus trabajos y actividades, hecho que permitió evidenciar su compromiso y motivación.

\section{CONSIDERACIONES FINALES}

Según los resultados se puede evidenciar que, en el área de la física, en contraste con el área de matemáticas, los estudiantes presentaron un avance mayor en su rendimiento académico. Una de las hipótesis planteadas por el grupo de investigadores de acuerdo al trabajo de campo realizado, y que puede dar lugar a una futura indagación, es que las prácticas de laboratorio virtual hacen que la física brinde un mayor acercamiento práctico a lo que se quiere enseñar, porque implica todo un proceso de investigación en el que incursiona el estudiante, es decir, el estudiante aprende desde un saber hacer donde utiliza una serie de procesos cognitivos y conceptuales para poner a prueba la aplicación o resolución de un problema (Losada y Moreno, 2003). En pocas palabras, la práctica en laboratorio por web, recurso con el cual cuenta Mate Club, permite relacionar en la praxis del estudiante sus saberes previos, así el estudiante va observando el proceso experimental.

Aquellos espacios digitales utilizados en Mate Club $^{4}$ para acceder a las simulaciones interactivas, fueron aquellos donde demostraron un mayor trabajo en equipo, el cuestionamiento, la inferencia y

4 En el área de física se trabajó laboratorios con PHET Interactive Simulations, y en el caso de matemáticas, se utilizó Geogebra, en la que tuvieron la posibilidad de visualizar interceptos, concavidad y puntos de inflexión de los diferentes tipos de funciones y relaciones cónicas. otras actitudes que son propias del trabajo científico. Tal como lo plantean Christian et al. (2003) citado por García et al. (2006), utilizar simulaciones interactivas supone un gran desarrollo en la enseñanza de la física porque además de permitir visualizar fenómenos que no son fáciles de abordar de otra manera, promueven un aprendizaje de conceptos y principios científicos basados en la investigación realizada por los estudiantes.

El diseño de la web Mate Club posibilitó llevar a cabo un proceso más participativo entre los agentes educativos involucrados y al mismo tiempo invitó a los estudiantes a aprender a utilizar herramientas digitales de recopilación de información como Examtime, para la elaboración y presentación de mapas conceptuales; Timeline, para la realización de líneas de tiempo; Pictochrad, para elaboración de infografías, entre otras. Muchos de estos trabajos realizados por los estudiantes se pueden apreciar en Mate Club.

La puesta en escena de Mate Club como estrategia pedagógica, llevó al aula una propuesta motivante y atrayente que no solo sirvió para mejorar en los estudiantes el nivel de comprensión de las temáticas abordadas de acuerdo con el plan curricular en las asignaturas de matemáticas y física, sino que también logró desarrollar las competencias necesarias para mejorar su rendimiento académico en el segundo período escolar.

Uno de los logros adicionales además de conseguir la mejora en el desempeño académico en las asignaturas abordadas fue haber encontrado en los estudiantes un cambio de actitud frente a las clases, la participación fue mucho más activa, la responsabilidad con los trabajos en el aula y la entrega de tareas se realizaron en los tiempos estimados. En otras palabras, todo el proceso de enseñanza y aprendizaje se cualificó a través de la apuesta de Mate Club apoyada desde la competencia digital del docente.

De igual manera la Web permitió a los estudiantes tener un acercamiento menos temeroso y atrayen- 
te a la mayoría de las temáticas abordadas en las asignaturas consideradas como difíciles e imposibles de aprender, lo que les hizo posible obtener mejores desempeños y, sobre todo, que se sintieran partícipes en la construcción de sus propios conocimientos, lo que afianzó su seguridad para exponer sus ideas, inquietudes y dificultades.

Pero todos los alcances académicos mencionados anteriormente deben involucrar un desafío para el docente. En términos de Villella (1996), implica que el maestro y el estudiante pongan en juego la creatividad pedagógica para dar respuestas satisfactorias a los problemas con contenido matemático presente no solo en el aula sino también en la cotidianidad.

Mate Club es ante todo un recurso de apoyo pedagógico para el profesor y el estudiante que requiera potenciar los conocimientos en matemática y física en los últimos niveles de enseñanza en la educación media. Sin embargo, se reconoce en el equipo investigador que aún hace falta y queda mucho camino por recorrer en cuanto a la búsqueda y formas de articular lo pedagógico con las TIC; es un campo que requiere ser explorado y divulgado para nutrir a otros colectivos académicos de experiencias valiosas y enriquecedoras que posibiliten afianzar los saberes y la praxis a los estudiantes que desean vivir y aventurarse al encantador y desafiante mundo de las matemáticas y la física.

\section{REFERENCIAS BIBLIOGRÁFICAS}

Arnau, Jaume, \& Bono, Roser. (2008). Estudios longitudinales de medidas repetidas: Modelos de diseño y análisis. Escritos de Psicología (Internet), 2(1), 3241. Recuperado en 02 de agosto de 2019, de http:// scielo.isciii.es/scielo.php?script $=$ sci_arttext\&pi$\mathrm{d}=$ S1989-38092008000300005\&lng=es\&tlng=es.

Arrieta, J. E. (2013). Las TIC y las matemáticas, avanzando hacia el futuro. Informe de grado de maestro en educación primaria. España: Universidad de Cantabria.
Bakia, M., Murphy, R., Anderson, K., \& Trinidad, G. (2011). International Experiences with Technology in Education: Final Report. Washington, D.C.: U.S. Department of Education. Documento mimeografiado

Calleja, R. (2010). Un modelo educativo para el siglo XXI. Comunidad escolar, 88. Recuperado de: http:// comunidad-escolar.pntic.mec.es/880/report1.html

Castro, S., Gusman, B. y Casado, D. (2007). Las Tic en los procesos de enseñanza y aprendizaje. Laurus, 13(23), 213-234. Recuperado de: http://www.redalyc.org/articulo.oa?id=76102311

García, A y Gil, M. R. (2006). Entornos constructivistas de aprendizaje basados en simulaciones informáticas. Revista Electrónica de Enseñanza de las Ciencias, 5(2), 304-322.

Hand, D. y Crowder, M. (1996). Practical longitudinal data analysis. London: Chapman \& Hall.

Hermosa Del vasto, P. M. (2015). Influencia de las tecnologías de información y comunicación (TIC) en el proceso enseñanza-aprendizaje: una mejora de las competencias digitales. Rev. Cient. Gen. José María Córdova, 13(16), 121-132.

Jiménez, C. (2018) ¿CUÁLES SON LAS CARRERAS CON MÁS FUTURO LABORAL? Revista Forbes de España. Recuperado de http://forbes.es/business/37068/cuales-las-carreras-mas-futuro-laboral/

Losada A. y Moreno H. (2003). Competencias básicas aplicadas al aula. Bogotá: Ediciones SEM.

Nieves, M. (1993). Actitudes hacia la matemática y rendimiento escolar. España: Isla de Tenerife

Pérez, A. S. (2010). Importancia de las TIC en la escuela. Revista para profesionales de la enseñanza, (7), 1-7.

Riveros, H. (1995). El papel del laboratorio en la enseñanza de la física en el nivel medio superior. Per- 
files Educativos, (68) Recuperado de http://www. redalyc.org/articulo.oa?id=13206806.

Ruiz S. J. (2008). Problemas actuales de la enseñanza aprendizaje de la matemática Revista Iberoamericana de Educación Universidad de Camagüey, Cuba. 47(3), 1-8.

The European Parliament and the Council of the European Union. (2006). On key competences for lifelong learning (2006/962/EC). Disponible (30/12/2006). Recuperado de: http:// eurlex.europa.eu/LexUriServ/LexUriServ.do?uri=OJ:L:2006:394:0010:0018:en:PDF

UNESCO (2008). Estándares de competencia en TIC para docentes. París: Unesco. Recuperado de: http:// eduteka.icesi.edu.co/pdfdir/UNESCOEstandaresDocentes.pdf

Vargas, G. (2015). Las TIC en la educación. Instituto pedagógico.

Villella, J. (1996). Sugerencias para la clase de Matemática. Argentina: AIQUE.

Vivancos, M. (2013). El futuro de la educación y las TIC.

Valencia, M. T., Serna, C. A., Ochoa, A. S., Caicedo, T. A., Monte, G. J. y Chávez, V. J. (2016). Competencias y estándares TIC desde la dimensión pedagógica: Una perspectiva desde los niveles de apropiación de las TIC en la práctica educativa docente. Santiago de Cali: Universidad Javeriana y Unesco

\section{LOS AUTORES}

María del Pilar Rodríguez Plaza: Licenciada en Pedagogía Infantil de la Institución Universitaria Antonio José Camacho. Integrante del Semillero de Investigación de las Tecnologías del Aprendizaje y del Conocimiento (SITAC)

Correo electrónico: pilarik0810@hotmail.com

Gloria Stella Zambrano Cerón: Licenciada en Pedagogía Infantil de la Institución Universitaria Antonio José Camacho. Integrante del Semillero de Investigación de las Tecnologías del Aprendizaje y del Conocimiento (SITAC)

Correo electrónico: gloriazn@gmail.com

Bryan Camilo Hernández: Psicólogo egresado de la Universidad San Buenaventura de Cali. Docente e investigador de la Facultad de Educación a Distancia y Virtual de la UNIAJC. Pertenece al Grupo de Investigación en Pedagogía (GIP) y Coordinador del Semillero de Investigación de las Tecnologías del Aprendizaje y del Conocimiento (SITAC) . Ha trabajado en líneas de investigación en evaluación y procesos pedagógicos.

Correo electrónico: bchernandez21@gmail.com 